Communications in Physics, Vol. 23, No. 2 (2013), pp. 185-191

\title{
SIMPLE MODEL FOR MARKET RETURNS DISTRIBUTION
}

\author{
CHU THUY ANH AND NGUYEN AI VIET \\ Institute of Physics, \\ Vietnam Academy of Science and Technology, \\ 10 Dao Tan, Ba Dinh, Hanoi, Vietnam \\ DO HONG LIEN \\ Ha Nam Teacher Training College, \\ Ly Thuong Kiet, Le Hong Phong Ward, Phu Ly City, \\ Ha Nam Province, Vietnam \\ E-mail: ctanh@iop.vast.ac.vn
}

Received 31 August 2012; revised manuscript received 25 April 2013 Accepted for publication 08 June 2013

\begin{abstract}
It has been observed that at the large time scales the distribution of stock market returns is convergent from Boltzmann distribution to Gaussian asymptotic one. To explain this universal phenomenon, we propose a new and simple dynamic model to describe this convergence by the time parameter in association with the introducing the concept of relaxation time for financial markets. The analysis of stock market data packages in different time intervals showed that our model fits well the financial market data. The meaning of so-called relaxation time has been qualitatively made clear, as a measure to estimate the stability of the market.
\end{abstract}

\section{INTRODUCTION}

It has been sixteen years[1] since the first time the term "econophysics" was introduced. Sixteen years is quite a long time for a man, but a short time for a oak, as well as for a new research branch. Although that, there have been more and more interests of physicists in econophysics. There are also several considerable successes of econophysicists[2], from both physics point of view and economic point of view.

Since both statistical mechanics and economics study macroscopic behaviours, i.e. collective modes, of big ensembles - collections of states of a physical system or that of market returns[3], and many phenomena found in statistical mechanics are able to be mapped into equivalent phenomena of economics and vice versa because of their common collective nature, the widely used approach in econophysics is statistical.

The fundamental law of equilibrium statistical mechanics is the Boltzmann distribution $e^{-E / k_{B} T}$, which measures probability for the distribution of states of the system. At a well-defined temperature $T$, Boltzmann distribution gives the probability that the system is in the corresponding energy state. Also temperature plays an important role, the higher temperature is, the crowder state the system is in. However, when a system is 
of equilibrium, temperature $T$ seems to have less effect on the shape of the distribution of the states. It is strongly believed that a Gaussian distribution is most naturally preferable for the stability of a system.

Analyzing a time series data in the distributions of returns on stock markets, econophysicists realized a transition from Boltzmann distribution to Gaussian one[4]. In a short time period, the distribution of returns could be approximated by Boltzmann distribution with high kurtosis, whereas for a longer time period, this distribution tends to converge to the Gaussian one with smoother peak and the probability for having big returns close to zero.

Some models based on the mathematical calculation[4], trying to describe this transition had been proposed. However, there still exist some missing links between the Boltzmann phase and the Gaussian one. That is why we propose in this paper a new simple model which is able to trace out the transition of market returns distribution. The model has been built based on detailed investigation of the NASDAQ database, and describes the distribution of market returns by time parameter in association with the introducing the concept of the relaxation time for a market.

The numerically obtained results showed that in despite of the simplicity of the model proposed, the model promises potential advantage of application as it fits well the real data.

\section{TIME EVOLUTION OF MARKET RETURNS DISTRIBUTION}

The logarithms of the stock prices $x(t)=\log p(t)$ and thus also NASDAQ 100, S\&P 500 , and DJIA indices have a special property: the minute returns $r(t)=\Delta x(t)$, i.e. the difference of price of a financial asset per minute, show an exponential distribution. This quantity $r(t)$ is one of the most used values in financial studies.

Take a look at the volatility of Dow Jones Industrial Average (DJIA) returns (2006/2011) (Fig. 1.), it could be easily remarked the relationship between returns fluctuation and financial crises.

That's why the transition of returns distributions is the objects of many econophysics studies[3, 5, 6, 7], including this paper.

In probability theory, the Gaussian distribution is a continuous probability distribution that is often used as a first approximation to describe real-valued random variables which tend to cluster around a single mean value. Theoretically, Gaussian distribution could be used to model returns distribution when studying the market for long time enough, also means, when the returns set is big enough. For a short time study, means for a small set of returns, this set of values could be considered as discontinuous one.

Boltzmann distribution is a certain distribution function or probability measure for the distribution of the states of a system. The shape of Boltzmann distribution is, in general, asymmetric, the maximum of probability is usually found at a non-zero value. There are some similar behaviours between returns distribution and Boltzmann one. Considering the returns in short time period, their values can be considered as discontinuous, and the most possible return value must difference from zero (if not, there is no gain no lost for every trading). For long time period study, return values set is much bigger, the values could be considered as continuous, the market tends to stable state, the return 


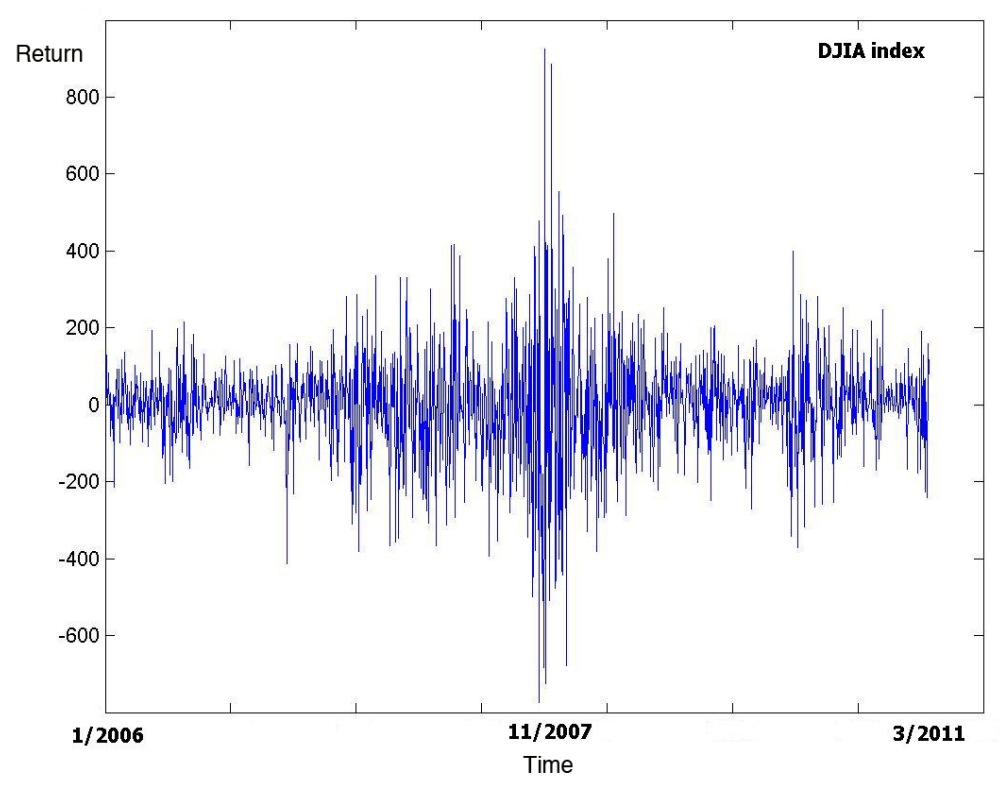

Fig. 1. Volatility of DJIA index returns from 2006 to 2011

varies around an average value. The distribution of market returns tends to converge to Gaussian distribution, as required by the central limiting theorem of statistical mechanics which states that the convolution of infinitely many arbitrary distribution functions of finite width always approaches a Gaussian distribution.

With real values data from DJIA and NASDAQ, distribution of returns has been shown as normal distribution.

Fig. 2 shows that DJIA index returns from 2007 to 2011 respect well Gaussian law, and Fig. 3 shows that returns in one day of Alcoa (Aluminum Company of America) evolves from Boltzmann distribution (30 minutes) to Gaussian one (4 hours).

This phenomenon is well observed in every trading studies. And in the next steps, we propose a simple model to describe it mathematically.

\section{SIMPLE MODEL FOR MARKET RETURNS DISTRIBUTION}

For short time period, the returns distribute following Boltzmann law

$$
P_{B}(r)=C_{B} e^{-|r| / T}
$$

where $P_{B}(r)$ is probability function that has the return $r$ in the asset of returns, $T$ is an effective temperature, and $C_{B}$ is normalization constant. In principle, there are different temperature $T_{ \pm}$for positive and negative returns, but to a good approximations we may equate both $T=T_{+}=T$. By definition, the probability function $P_{B}$ should be normalized 


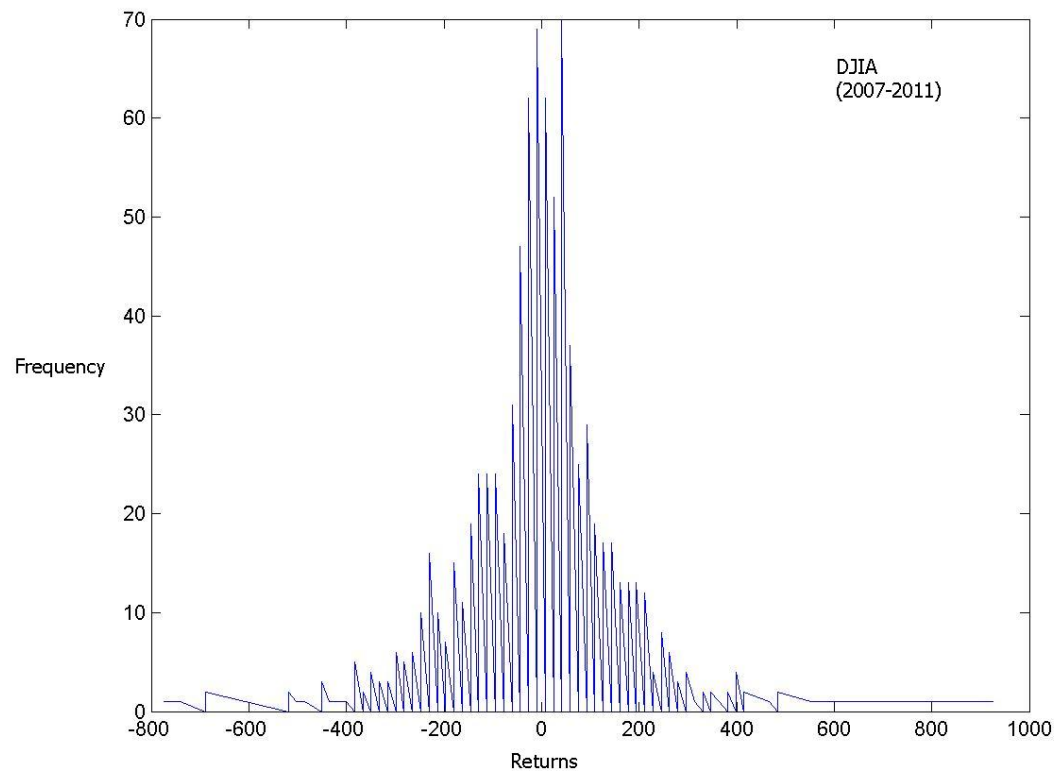

Fig. 2. Frequencies of DJIA index returns from 2007 to 2011

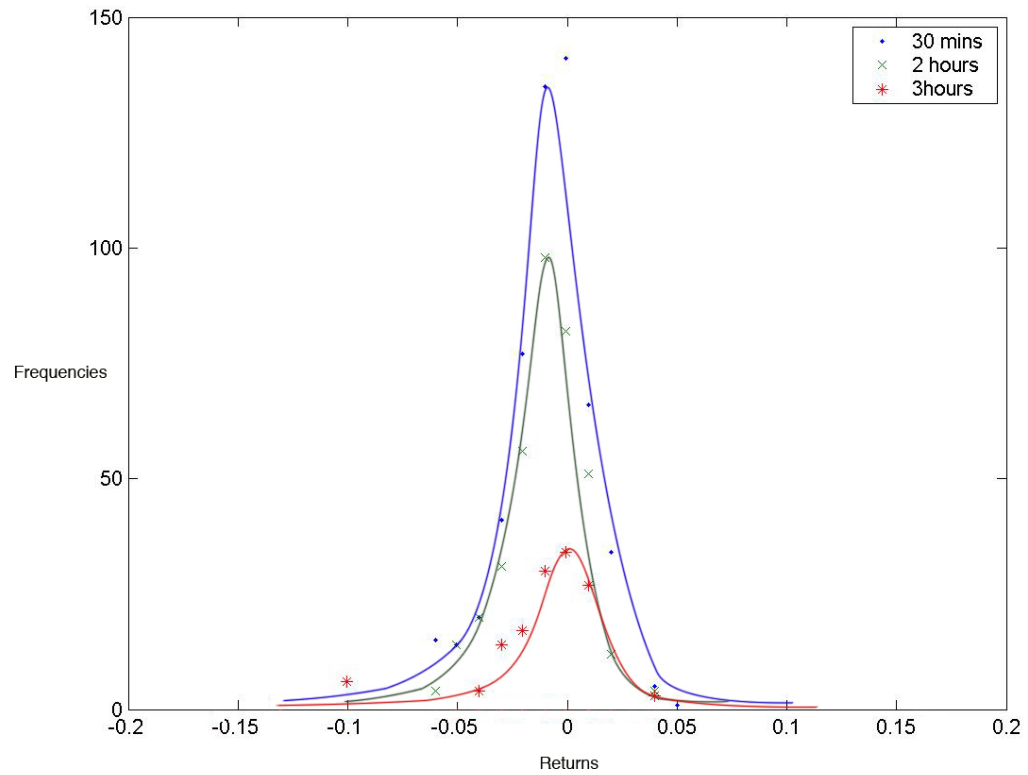

Fig. 3. Distributions of Alcoa returns in one day (2011) 


$$
\int_{-\infty}^{+\infty} P_{B}(r) d r=1
$$

determining the normalization constant $C_{B}$, for the symmetric Boltzmann distribution, given above,

$$
C_{B}=\frac{1}{2 T}
$$

After a long period of time, which depends on many other parameters, the returns re-distribute following the Gaussian distribution

$$
P_{G}(r)=C_{G} e^{-r^{2} / \sigma^{2}}
$$

where $P_{G}$ is probability to have the return $r$ in the asset of returns, $\sigma^{2}$ is the variance, and $C_{G}$ is normalization constant. The normalized expression for Gaussian distribution

$$
\int_{-\infty}^{+\infty} P_{G}(r) d r=1
$$

determines the normalization constant of the given Gaussian distribution

$$
C_{G}=\frac{1}{\sqrt{2 \pi} \sigma} .
$$

Based on the fact that at the initial time, i.e. $t$ is close to 0 , returns distribute following Boltzmann law, and at some final time, when $t$ gets sufficiently large, the distribution of returns tends to converge to Gaussian one, we propose the following model for the time evolution of the distributions

$$
P(r, t)=P_{B} u(t)+P_{G} v(t),
$$

The normalization condition for the distribution is that

$$
\int_{-\infty}^{+\infty} P(r, t) d r d t=1
$$

where the functions $u(t)$ and $v(t)$ describe time dependence of probability function $P(r, t)$, and are chosen as

$$
\left\{\begin{array}{ll}
t=0 & : u(0)=1, v(0)=0 \\
t \rightarrow \infty & : u(\infty)=0, v(\infty)=1
\end{array} .\right.
$$

The simplest representation of the time dependent functions $u(t)$ and $v(t)$ that satisfies the above condition is

$$
\left\{\begin{array}{l}
u(t)=e^{-t / \tau} \\
v(t)=1-e^{-t / \tau},
\end{array},\right.
$$

where newly introduced parameter $\tau$ is the relaxation time which is the characteristic time for the evolution. For a concrete observation, the value of its relaxation time is directly 
related to market stability. Here, we did bring the concept of relaxation time which is well developed for dynamic evolution in physics, into economic study. In the context, the relaxation time would be understood as a measure of dynamic behaviours of the stock markets.

Inserting the expression of the functions $u(t)$ and $v(t)$ into model distribution (7), the probability function $P(r, t)$ now reads

$$
P(r, t)=C_{B} e^{-|r| / T} e^{-t / \tau}+C_{G} e^{-r^{2} / \sigma^{2}}\left(1-e^{-t / \tau}\right) .
$$

Obviously, the expression (11) reflects the time evolution of the time dependent returns distribution $P(r, t)$ as expected.

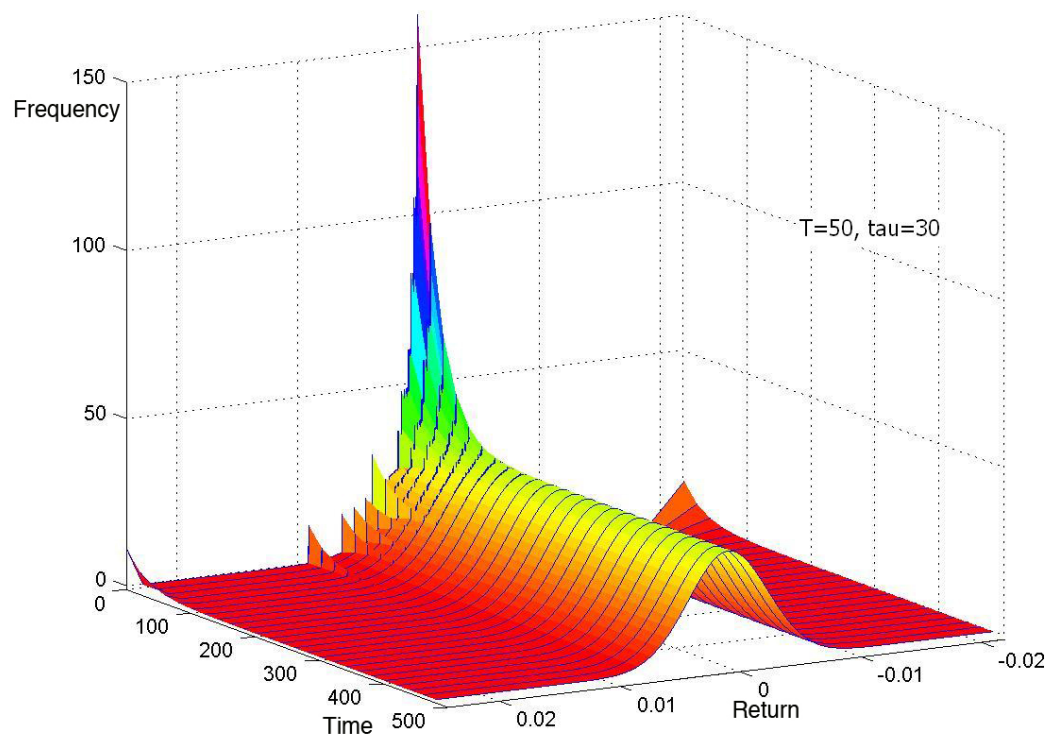

Fig. 4. Distribution of DJIA returns in 1801 days (2009-2011)

Analysing returns in DJIA time-series data package shows that daily DJIA returns distribution, theoretically and practically, moves from Boltzmann to Gaussian one in the time evolution of observation. Moreover, the numerical calculation based on our model distribution $P(r, t)$ gives a step further, allowing to obtain an approximate value of relation time for the dynamic process under consideration, in the case of the DJIA index, $\tau=30$.

\section{CONCLUSION AND DISCUSSION}

The model has been built and verified based on the database of NASDAQ and Dow Jones Industrial Average[10, 11]. The modeling result fits well with real values distribution.

There has been introduced three measurable parameters: effective temperature $T$, variance $\sigma$ and relaxation time $\tau$ which help to quantify the chaotic state of the market. 
Variance $\sigma$ can be also considered as the market stability factor. The model will provide a simple and useful tool for the financial analyst.

This paper has just presented the simplest model to describe market fluctuation. In fact, financial returns are known to be non-gaussian and exhibit fat-tailed distribution $[8,9]$. In the next publication, we will evaluate the model and build the theory for it to have a better description on market fluctuations.

\section{REFERENCES}

[1] A. Carbone, G. Kaniadakis and A.M. Scarfone Physica A 382 (2007).

[2] Victor M. Yakovenko, J. Barkley Rosser, Jr, Rev. Mod. Phys., 81, 1703 (2009)

[3] A. Dragulescu and V. M. Yakovenko, Eur. Phys. J. B., 17, 723-729 (2000)

[4] H. Kleinert and X. J. Chen, Physica A 383 (2007)

[5] A. Christian Silva and Victor M. Yakovenko, Physica A 324 (2003)

[6] Fei Ren and Wei-Xing Zhou, New J. Phys 12 (2010)

[7] Raj Kumar Pan and Sitabhra Sinha Phys. Rev. E, 76046116 (2007)

[8] Naoki Kozuki, Nobuko Fuchikami, Physica A 329 (2002)

[9] R. Friedrich, J. Peinke, Ch. Renner, Phys. Rev. Lett 84 (2000)

[10] www.nasdaq.com

[11] www.djaverages.com 